\section{Neurological Manifestations of Hereditary Haemorrhagic Telangiectasis}

British Medical Fournal, 1970, 3, 688

This communication emphasizes the neurological significance of pulmonary lesions in hereditary haemorrhagic telangiectasis and illustrates the beneficial effects of chest surgery on neurological symptoms.

\section{CASE REPORT}

A 19-year-old laboratory technician noticed dyspnoea and pleuritic chest pain shortly after playing football. Two days later while at rest he suddenly experienced a feeling of unreality and his right hand became numb and movements were clumsy. Subsequently his left hand was similarly affected. This episode lasted about five minutes and was followed by severe headache and a small haemoptysis. He had similar attacks on two days subsequently. During the second attack he lost consciousness and was admitted to hospital.

$\mathrm{He}$ had suffered from frequent epistaxes since early childhood and described four episodes of haemoptysis during the previous 18 months. He had also experienced occasional episodes of blurred vision, but it was not certain whether these were associated with haemoptyses. His great-grandmother suffered from frequent "bleeding from the finger-tips," his grandfather had frequent epistaxes which required blood transfusions, and his mother had a similar illness (see below).

On admission he was deeply unconscious, had fixed dilated pupils, and blood was oozing from his mouth. After intubation and bronchial lavage he recovered gradually, though jerking movements persisted in his right arm during the next two days. Haematological investigations showed no abnormality. Chest $x$-ray examination showed patchy consolidation at the left mid and lower zones; skull $x$-ray film was negative. Lumbar puncture produced clear fluid at normal pressure containing $75 \mathrm{mg}$. of protein $/ 100$ $\mathrm{ml}$. He was found to be alert, well-orientated, and left-handed. No abnormalities were found other than a discrete red telangiectasis $2 \mathrm{~mm}$. in diameter on the tongue, four telangiectases on the lower lip, and three on the dorsum of the right hand.

During the next three months he had 15 further episodes in which he experienced generalized "tingling," often associated with weakness of the right side. During five of these attacks he lost consciousness, in four there was an associated feeling of tightness in the chest, and in five he had a haemoptysis. He was treated with anticonvulsants, and further investigations were performed. A pulironary scintillation scan showed diminished uptake at the iu - ion of the upper and middle thirds of the left lung. PulmoI' : arteriography showed normal main pulmonary arteries, but the we was a large arteriovenous malformation in the apical segment of the left lower lobe and another in the anterior segment of the left upper lobe, and a minute malformation in the left upper lobe and another in the left lower lobe. There were no major lesions in the right lung field. It was believed that the neurologica! symptoms were related to the large pulmonary vascular lesions, which are accepted features of hereditary haemorrhagic telangiectasis (Hodgson et a!., 1959). At thoracotomy the apical segment of the left lower lobe, containing a large localized arteriovenous malformation, and two subpleural lesions were resected. Postoperatively he was given phenobarbitone $50 \mathrm{mg}$. t.d.s., but as he had no further fits this therapy was stopped three months later. He remained well with no further fits or haemoptyses.

\section{Comment}

In hereditary haemorrhagic telangiectasis focal neurological manifestations may be either secondary to pulmonary lesions or due to arteriovenous 'malformations in the brain. In this patient it is improbable that a primary intracranial lesion was responsible for his symptoms because a normal carotid arteriogram excluded a large malformation and pin-head telangiectases in the brain or meninges are almost always silent (Boczko, 1964). Recurrent intracerebral emboli are therefore the more likely cause of his symptoms, and the frequent simultaneous occurrence of haemoptyses-often with frothy blood-suggests air embolism. The complete absence of neurological symptoms for one year after thoracotomy is further evidence that the lung lesions were responsible for his fits.

The patient's mother had suffered from frequent epistaxes since the age of 13 and had several telangiectases on the lower lip. In 1950 she suffered a right hemiplegia of sudden onset which slowly resolved over the subsequent two years. The cause of the hemiplegia was not clear. In 1952 she was investigated because of haemoptysis, and a pulmonary arteriovenous malformation with a considerable shunt was found. After a left lower lobectomy she made an uneventful recovery and had no further haemoptyses or neurological manifestations. In retrospect it seems probable that her hemiplegia was due to an embolus secondary to her pulmonary lesion. The coexistence of a cerebral malformation was unlikely. We have been able to find only two reports of patients with both lesions (Boczko, 1964; Warren, 1969), though there is information about pulmonary and cerebral malformations occurring in different members of the same family (Chandler, 1965).

The present patients were symptom-free for 1 and 17 years respectively after operation. Their histories draw attention to the importance of considering a pulmonary cause for the neurological manifestations of hereditary haemorrhagic telangiectasis, even when ordinary chest $x$-ray pictures are normal (Bergqvist et al., 1962), and illustrate the successful role of thoracic surgery.

We would like to thank Mr. I. McCaul and Dr. G. Stern for their advice during the preparation of this report on their patient.

I. M. STANLEY, M.B., M.R.C.P.

K. R. HUNTER, M.B., M.R.C.P.

Ncurological Unit, University College Hospital, London W.C.1

\section{REFEPENCES}

Bergqvist, N., Hessen, I., and Hey, M. (1962). Acta Medica Scandinavica, 171, 301 .

Boczko, M. L. (1964). Fournal of Nervous and Mental Diseases, 139, 525 .

Chandler, D. (1965). Archives of Internal Medicine, 116, 277.

Hodgson, C. H., Burchell, H. B., Good, C. A., and Clagett, O. T. (1959). New England 7ournal of Medicine, 26i, 625 Warren, G. C. (1969). Fournal of Neurosurgery, 30, 618. 